\title{
Antidot tunneling between Quantum Hall liquids with different filling factors
}

\author{
V.V. Ponomarenko* and D.V. Averin \\ Department of Physics and Astronomy, University of Stony Brook, SUNY, Stony Brook, NY 11794
}

(Dated: November 20, 2018)

\begin{abstract}
We consider tunneling through two point contacts between two edges of Quantum Hall liquids of different filling factors $\nu_{0,1}=1 /\left(2 m_{0,1}+1\right)$ with $m_{0}-m_{1} \equiv m>0$. Properties of the antidot formed between the point contacts in the strong-tunneling limit are shown to be very different from the $\nu_{0}=\nu_{1}$ case, and include vanishing average total current in the two contacts and quasiparticles of charge $e / m$. For $m>1$, quasiparticle tunneling leads to non-trivial $m$-state dynamics of effective flux through the antidot which restores the regular "electron" periodicity of the current in flux despite the fractional charge and statistics of quasiparticles.
\end{abstract}

PACS numbers: 73.43.Jn, 71.10.Pm, 73.23.Ad

One of the most interesting features of the Fractional Quantum Hall Liquids (FQHLs) is the existence of quasiparticles with fractional charge 1] and exchange statistics 2]. Although the quasiparticles are defined most simply in the incompressible bulk of the FQHL, in the lowenergy transport experiments, quasiparticles are created typically at the liquid edges, e.g. by tunneling between them. In the simplest case of FQHL with the filling factor $\nu=1 /$ odd, the quasiparticles that tunnel through the liquid between its edges coincide with the quasiparticles in the bulk 3] and their fractional charge $\nu e$ can be measured experimentally [4, 5]. So far, fractional statistics of quasiparticle has not been directly observed in experiments, although there is experimental $[\underline{6}$ and theoretical 7] interest to manifestations of this statistics in the noise correlators of the tunnel currents.

Strong tunneling between edges of FQHLs with different filling factors should create quasiparticles which are different from those in the bulk of the liquids but still have fractional charge and statistics [8, 9]. Untill now, such tunneling has been considered only in the geometry of a single point contact [8] or multiple contacts 9] for which the interference between different contacts is not important (i.e., the edges do not form closed loops). The purpose of this work is to study an "antidot" tunnel junction: two separate point contacts at points $x_{1}$, $x_{2}$ along the $x$-axis between two single-mode edges of QHLs with different filling factors $\nu_{0,1}=1 /\left(2 m_{0,1}+1\right)$ with $m_{0}>m_{1} \geq 0$ - see Fig. 1 . In this geometry, the tunneling processes at two point contacts interfere and statistics of tunneling quasiparticles directly affects the dc current.

If the two filling factors are equal, $\nu_{0}=\nu_{1} \equiv \nu$, as in experiments [4], strong tunneling leads to formation of a closed edge between the points $x_{1}$ and $x_{2}$ encircling the antidot and separated from external edges of the surrounding uniform QHL [10]. Quasiparticles of charge $\nu e$ can then tunnel between the external edges

\footnotetext{
*on leave from St. Petersburg State Polytechnical University, Cen-
} ter for Advanced Studies, St. Petersburg 195251, Russia. through the antidot. As shown below, the situation is very different for $\nu_{0} \neq \nu_{1}$, and the antidot formed between the point contacts does not decouple completely from external edges even in the limit of strong tunneling. As a result, the total tunnel current between the two QHLs vanishes in this limit. Also, interference between the two contacts produces the quasiparticles of charge $e / m$ determined by the change $m$ of the number of flux pairs attached to the electrons 11] in the two liquids: $m=m_{0}-m_{1}$. In general, proper account of the flux attachement to tunneling electrons is one of the key elements of the description of strong multi-point tunneling between QHLs with different filling factors. Changes in the number of attached flux pairs should be accounted for by inclusion of appropriate statistical contributions into the bosonic tunneling fields describing different point contacts [12. These statistical terms do not affect the perturbative expansions in tunneling, but make the tunneling fields at different point contacts commute, the feature that becomes important in the limit of strong tunneling.

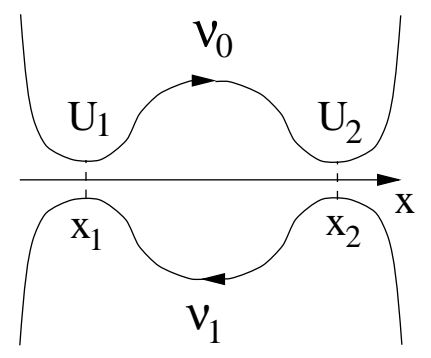

FIG. 1: "Antidot" junction considered in this work: two point contacts with tunneling amplitudes $U_{j}$ formed at points $x_{j}$, $j=1,2$, between two counter-propagating edges of QHLs with different filling factors $\nu_{0}$ and $\nu_{1}$. The edges are assumed to support one bosonic mode each, with arrows indicating direction of propagation of these modes.

Quantitatively, we describe the antidot junction (Fig. 1) between two single-mode edges with filling factors $\nu_{l}=1 /\left(2 m_{l}+1\right), l=0,1$, by adopting the standard bosonization approach in which the electron operator $\psi_{l}$ 
of the edge $l$ is expressed as 13

$$
\psi_{l}=\left(D / 2 \pi v_{l}\right)^{1 / 2} \xi_{l} e^{i(-1)^{l}\left[\phi_{l}(x, t) / \sqrt{\nu_{l}}+k_{l} x\right]} .
$$

Here $\phi_{l}$ are the two bosonic modes propagating in the opposite direction with velocities $(-1)^{l} v_{l}, v_{l}>0$, the Majorana fermions $\xi_{l}$ account for mutual statistics of electrons in different edges, and $D$ is a common energy cut-off of the edge modes. The Fermi momenta $k_{l}$ correspond to the average electron density in the edges, while the operators of the density fluctuations are: $\rho_{l}(x, \tau)=\left(\sqrt{\nu_{l}} / 2 \pi\right) \partial_{x} \phi_{l}(x, \tau)$. Bosonic fields $\phi_{l}$ have the standard quadratic Lagrangian defined by the Fourier transform of the imaginary-time-ordered correlators (see, e.g., [9]) $\left\langle\phi_{l}(x) \phi_{p}\right\rangle=\delta_{l p} g\left((-1)^{l} x / v_{l}, \omega\right)$, where

$$
g(z, \omega)=\frac{2 \pi}{\omega} \operatorname{sgn}(z)\left(-\frac{1}{2}+\theta(\omega z) e^{-\omega z}\right),
$$

which in particular imply the usual equal-time commutation relations $\left[\phi_{l}(x), \phi_{p}(0)\right]=i \pi \operatorname{sgn}(x) \delta_{l p}(-1)^{l}$.

With the bosonized electron operators, Langrangian describing electron tunneling in the two contacts is:

$$
\mathcal{L}_{t}=\sum_{j=1,2}\left[\frac{D U_{j}}{2 \pi} e^{i \kappa_{j}} e^{i \lambda \varphi_{j}}+\text { h.c. }\right] \equiv \sum_{j=1,2}\left(T_{j}^{+}+T_{j}^{-}\right),
$$

where $U_{j}$ and $\kappa_{j}$ are the absolute values and the phases of the dimensionless tunneling amplitudes, and

$$
\lambda \varphi_{j}(t) \equiv \frac{\phi_{0}\left(x_{j}, t\right)}{\sqrt{\nu_{0}}}+\frac{\phi_{1}\left(x_{j}, t\right)}{\sqrt{\nu_{1}}}, \quad \lambda=\left[\frac{\nu_{0}+\nu_{1}}{\nu_{0} \nu_{1}}\right]^{1 / 2} .
$$

The factor $\lambda$ is chosen in such a way that the normalization of the bosonic operators $\varphi_{j}$ coincides with that of the fields $\phi_{l}$, so that the imaginary-time correlators of $\varphi_{j}$ follow directly from Eq. (11). The products of the Majorana fermions $\xi_{1} \xi_{2}$ were omitted from the Lagrangian (2), since they at most can produce an irrelevant overall constant shift of the phases $\kappa_{j}$. These phases include contributions from the external magnetic flux $\Phi$ through the antidot and from the average electron numbers $N_{0,1}$ on the two sides of its perimeter, so that $\kappa_{2}-\kappa_{1}=2 \pi\left[\left(\Phi / \Phi_{0}\right)+\left(N_{0} / \nu_{0}\right)+\left(N_{1} / \nu_{1}\right)\right]+$ const $\equiv \kappa$, where $\Phi_{0}=h / e$ is the "electron" flux quantum.

If the bias voltage $V$ is applied to the junction, the electron current operator is $I^{e}=i \sum_{j=1,2} \sum_{ \pm}( \pm) T_{j}^{ \pm} e^{\mp i V t}$. Its average includes contributions from the individual point contacts $\bar{I}_{j}^{e}$ and the phase-sensitive interference term $\Delta I^{e}(\kappa):\left\langle I^{e}\right\rangle=\sum_{j} \bar{I}_{j}^{e}+\Delta I^{e}(\kappa)$. At temperature $T$, in the lowest non-vanishing order of the perturbation theory in $U_{j}$, the two contributions are:

$$
\bar{I}_{j}^{e}=\left(U_{j}^{2} D / 2 \pi\right)(2 \pi T / D)^{\lambda^{2}-1} C_{\lambda^{2}}(V / 2 \pi T),
$$

where $C_{g}(v) \equiv \sinh (\pi v)|\Gamma(g / 2+i v)|^{2} /[\pi \Gamma(g)]$, and

$$
\Delta I^{e}=\left(\frac{2 U_{1} U_{2} D}{\pi}\right)\left(\frac{2 \pi T}{D}\right)^{\lambda^{2}-1} \operatorname{Im}\left\{\int_{-\infty}^{\infty} d s \sin \left(\kappa-\frac{s V}{\pi T}\right)\right.
$$

$$
\left.\cdot \prod_{l=0,1}\left[i \sinh \left(s-(-1)^{l} t_{l} \pi T-i 0\right)\right]^{-1 / \nu_{l}}\right\}
$$

Here $t_{l}$ is the time of electron propagation between the two point contacts along the $l$ th edge.

Equations (31) and (4) show that at low energy, when $V, T<\Delta$, where $\Delta=t_{\Sigma}^{-1}, t_{\Sigma} \equiv t_{0}+t_{1}$, the terms $t_{l} \pi T$ can be omitted in Eq. (4), and the full current $\left\langle I^{e}\right\rangle$ is given by Eq. (3) with the tunnel amplitude $U_{j}$ replaced by the coherent sum of the two point-contact amplitudes: $U_{j} \rightarrow\left|U_{1} e^{i \kappa_{1}}+U_{2} e^{i \kappa_{2}}\right|$. This implies that similarly to the situation of one point contact, the total electron tunneling current $\left\langle I^{e}\right\rangle$ is suppressed at low energies $E$ roughly as $E^{\lambda^{2}-1}$, where $E \simeq \max (V, T)$. At large energies, the total current $\left\langle I^{e}\right\rangle$ is dominated by the contributions (3) from individual point contacts, with interference term (4) suppressed exponentially at large temperatures $T>\Delta$, or growing slower that the individual currents (31) with increasing voltage $V>\Delta>T$. Therefore, if $\Delta<T_{X}$, transition from weak to strong tunneling occurs at the same energy scale $T_{X} \simeq D U^{-2 /\left(\lambda^{2}-2\right)}$ (we assume for simplicity that $U_{1} \simeq U_{2} \equiv U$ ) as for one point contact [14, 15], with the perturbative result for electron tunneling valid at $T, V<T_{X}$. As shown below, if $\Delta>T_{X}$, electron tunneling become strong and perturbation theory in $U$ is not correct.

The main focus of our work is on this strong-tunneling limit that can be realized if $U \gg 1$. In this case, the tunneling Lagrangian (2) gives the dominant part of the action and a natural approach to solving the systems dynamics would be to fix the tunneling modes $\varphi_{j}$ at the extrema of the Lagrangian (2). This, however, can not be done directly since the operators $\varphi_{j}$ at two point contacts do not commute: $\left[\varphi_{1}, \varphi_{2}\right]=i \pi\left(\nu_{1}-\nu_{0}\right) /\left(\nu_{1}+\nu_{0}\right)$, and can not be simultaneously fixed, although the different transfer terms $T_{j}^{ \pm}$(2) still commute among themselves, e.g.,

$$
T_{1}^{ \pm} T_{2}^{ \pm}=e^{2 \pi m i} T_{2}^{ \pm} T_{1}^{ \pm} .
$$

This problem can be resolved using the fact that the tunneling Lagrangian (2) does not uniquely define the strong-tunneling limit. The factors $\exp \left\{ \pm i \sqrt{2 m} \eta_{j}\right\}$ with free zero-energy bosonic modes $\eta_{j}$ defined by their imaginary-time-ordered correlators: $\left\langle T_{\tau} \eta_{i}(\tau) \eta_{j}(0)\right\rangle=$ $i \pi \Theta((j-i) \tau)\left(1-\delta_{i j}\right)$, can be included [12] into the terms $T_{j}^{ \pm}$in (2) without changing the perturbation expansion of the partition function in $\mathcal{L}_{t}$ in any order. The new tunneling operators $\varphi_{j}+\sqrt{2 m} \eta_{j}$ commute, and can be fixed by the natural strong-tunneling conditions:

$$
\lambda \varphi_{j}+\sqrt{2 m} \eta_{j}+\kappa_{j}=2 \pi n_{j} .
$$

Transport properties of the antidot junction (Fig. 1) can be described then as successive transformations of the fields $\phi^{i n}\left(x_{j}\right)$ entering individual contacts into the 
outgoing fields $\phi^{\text {out }}\left(x_{j}\right)$ : $\phi^{\text {out }}\left(x_{j}\right)=\hat{P} \phi^{\text {in }}\left(x_{j}\right)$, where $[$, 9]

$$
P_{00}=-P_{11}=\frac{\nu_{0}-\nu_{1}}{\nu_{0}+\nu_{1}} ; \quad P_{01}=P_{10}=-\frac{2 \sqrt{\nu_{0} \nu_{1}}}{\nu_{0}+\nu_{1}},
$$

and $\phi^{\text {in, out }}\left(x_{j}\right)=\left(\phi_{0}\left(x_{j} \mp 0\right), \phi_{1}\left(x_{j} \pm 0\right)\right)^{T}$. The transformations (7) at the two contacts should be combined according to the edge propagation diagram shown in Fig. 2 , which involves multiple interferences along the closed loop formed between the points $x_{1}$ and $x_{2}$. Summation over these interferences gives a very simple final result $\phi^{\text {out }}=\phi^{\text {in }}$ which means that the fields and the currents incident on the junction remain the same in the outgoing edges, and the total tunnel current between the two QHL vanishes.

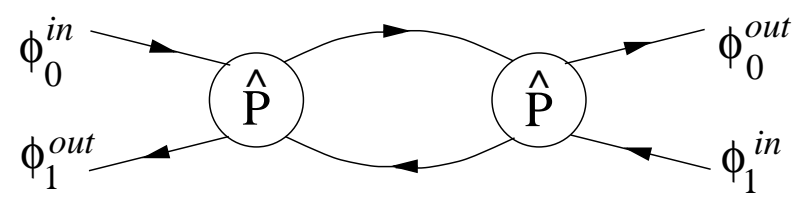

FIG. 2: Diagram of the strong-coupling edge propagation in the antidot junction.

This discussion assumes that large tunnel amplitudes $U_{j}$ completely suppress quantum fluctuations of the fields $\varphi_{j}$. For large but finite $U_{j}$, the fields can tunnel between different minima $n_{j}$ of the strong-coupling solution (6), the process that represents quasiparticle backscattering and can be described quantitatively in terms of the instanton expansion [9]. The operators $\left(W_{j} D / 2 \pi\right) \exp \left\{ \pm i 2 \theta_{j} / \lambda\right\}$, where $\theta_{j}$ are dual to $\varphi_{j}$ : $\left[\theta_{j}, \varphi_{k}\right]=i \pi(-1)^{j} \delta_{j k}$, generate the jumps of $\varphi_{j}$ changing $n_{j}$ by $\mp(-1)^{j}$. Instanton tunneling amplitude $W_{j}$ can be estimated as in one contact, $W_{j} \simeq U_{j}^{\left[\left(2 / \lambda^{2}\right)-1\right] /\left[\left(\lambda^{2} / 2\right)-1\right]}$ [16]. Using the commutation relations of the edge fields $\phi_{l}$ one can express $\theta_{j}$ in terms of $\phi_{l}$ :

$$
\theta_{j}=(-1)^{j+1}\left[\phi_{0}\left(x_{j}-0\right) / \sqrt{\nu_{0}}+\phi_{1}\left(x_{j}+0\right) / \sqrt{\nu_{1}}\right] / \lambda,
$$

and see that as in the case of one point contact [15], instanton tunneling operators transfer charge $\pm(-1)^{j} 2 \nu_{0} \nu_{1} /\left(\nu_{0}+\nu_{1}\right)$ from $\mathrm{QHL}_{\nu_{1}}$ into the $\mathrm{QHL}_{\nu_{0}}$. In the antidot junction, however, these charges undergo further scattering in accordance with the diagram in Fig. 2. Accounting for this scattering with the help of Eq. (77) we see that the total quasiparticle charge transferred from $\mathrm{QHL}_{\nu_{1}}$ into the $\mathrm{QHL}_{\nu_{0}}$ by each instanton tunneling is $\pm 1 / m$.

Evaluating action on the instanton trajectories similarly to what is done in [9], we obtain the Lagrangian $\overline{\mathcal{L}}_{t}$ for quasiparticle tunneling that has the form dual to the Lagrangian (2):

$$
\overline{\mathcal{L}}_{t}=\sum_{j=1,2}\left[\frac{W_{j} D}{2 \pi} \bar{F}_{j} \exp \left\{i\left(\frac{\kappa_{j}}{m}+\frac{2 \theta_{j}}{\lambda}-\frac{V t}{m}\right)\right\}+h . c .\right]
$$

$$
\equiv \sum_{j=1,2} \sum_{ \pm} \bar{T}_{j}^{ \pm} e^{\mp i V t / m}
$$

The correlators of the fields $\theta_{j}$ in (9) can be found from Eq. (8) combined with the strong-coupling scattering scheme described by Fig. 2 and Eq. (7):

$$
\begin{gathered}
\left\langle\theta_{j} \theta_{j}\right\rangle=g(0, \omega)+\frac{1}{2} \sum_{n=1}^{\infty} P_{01}^{2 n} \sum_{ \pm} g\left( \pm n t_{\Sigma}, \omega\right), \\
\left\langle\theta_{1} \theta_{2}\right\rangle=\sum_{n=0}^{\infty} \frac{P_{01}^{2 n}}{\nu_{0}+\nu_{1}}\left[\nu_{1} g\left(t_{0}+n t_{\Sigma}, \omega\right)+\nu_{0} g\left(-t_{1}-n t_{\Sigma}, \omega\right)\right] .
\end{gathered}
$$

The unitary operators $\bar{F}_{j}$ in (9) are Klein factors describing statistics of quasiparticles and are characterized by the following relations:

$$
\bar{F}_{1} \bar{F}_{2}=e^{\frac{2 \pi i}{m}} \bar{F}_{2} \bar{F}_{1}, \quad\left\langle\bar{F}_{1}^{k}\left(\bar{F}_{1}^{+}\right)^{p} \bar{F}_{2}^{l}\left(\bar{F}_{2}^{+}\right)^{q}\right\rangle=\delta_{k p} \delta_{l q},
$$

were the Kronecker symbol $\delta_{i j}$ is defined modulo $m$. These relations originate from the effective flux through the antidot which includes external magnetic flux and statistical contribution and is equal to $n=n_{1}-n_{2}$ (in units of $\left.\Phi_{0}\right)$ in the strong-tunneling limit (6). Because of the interference in the two point contacts, tunneling amplitudes of quasiparticles of charge $1 / \mathrm{m}$ acquire the phases $e^{ \pm i 2 \pi n / m}$ which distinguish $m$ states of the antidot with different fluxes $n^{\prime} \neq n \bmod (m)$. Since these states are equivalent in all other respects, summation of the tunneling amplitudes over them gives rise to the second relation in (11). On the other hand, each quasiparticle tunneling changes $n$ by \pm 1 , the fact accounted for by the first part of Eq. (11), which also ensures commutativity of the transfer operators $\bar{T}_{j}^{ \pm}$in (91). In addition, electron commutation relations (5) mean that each electron tunneling changes the effective flux between the two tunneling points $x_{1,2}$ by $m$, and the variation of the flux by \pm 1 due to quasiparticle tunneling can be seen as the basic reason for the fractional quasiparticle charge $1 / \mathrm{m}$.

From Lagrangian (9), the operator $I$ of the quasiparticle current is: $I=(i / m) \sum_{j=1,2} \sum_{ \pm} \pm \bar{T}_{j}^{ \pm} e^{\mp i V t / m}$. Similarly to electron current (3), (4), its average contains contributions from individual point contacts and phasesensitive interference term: $\langle I\rangle=\sum_{j} \bar{I}_{j}+\Delta I(\kappa)$. Since in the absence of backscattering the current between the two QHLs vanishes in the strong-tunneling limit, quasiparticle backscattering gives the full current. Behavior of this current depends on the relation between the crossover energy $T_{X}$ and the antidot "quantization" energy $\Delta$. If the two point contacts are far apart and/or the tunneling amplitudes $U_{j}$ are sufficiently small, then $\Delta<T_{X}$, and the transition from weak to strong tunneling occurs at large energies at which the interference is already suppressed, so that this transition has the same form as in one point contact [14, 15]. In particular, at 
energies above $T_{X}$, the lowest-order perturbation theory in quasiparticle tunneling (9) gives:

$$
\bar{I}_{j}=\frac{1}{m}\left(W_{j}^{2} D / 2 \pi\right)\left(\frac{2 \pi T}{D}\right)^{4 / \lambda^{2}-1} C_{4 / \lambda^{2}}(V / 2 \pi T m) .
$$

The current (12) is carried by individual quasiparticles of charge $1 / m$, which should be seen at $V \gg T$ in the shot noise caused by the flow of this current.

If the two point contacts are close and/or $U_{j}$ 's are large, $\Delta>T_{X}$, and electron interference in the two contacts becomes important. Renormalized electron tunneling amplitude for the current encircling the antidot can be estimated roughly as $U(\Delta / D)^{\lambda^{2}-1}$ and becomes large when $\Delta>T_{X}$. In this regime, dynamics of tunneling should be discussed in terms of quasiparticle backscattering even at low energies $V, T$. The form of transition from weak to strong interference at $\Delta \simeq T_{X}$ depends on $m$ which controls the energy scaling of the quasiparticle amplitudes $W_{j}$ at low energies. As one can see from the correlators (10) of the fields $\theta_{j}$, the scaling dimensions of $W_{j}$ at energies above and below $\Delta$ are equal to $2 / \lambda^{2}-1$ and $\lambda^{2} / 2 m^{2}-1$, respectively. In the case of one-flux-pair variation, $m=1$, (i.e., $\nu_{0}=1 / 3$ and $\nu_{1}=1$, or $\nu_{0}=1 / 5$ and $\nu_{1}=1 / 3$ ) both the quasiparticle charge and the low-energy scaling dimension $\lambda^{2} / 2-1$ of tunneling amplitudes coincide with those for electrons. This means that for $T_{X}<\Delta$ the quasiparticle current is perturbative in $W$ at any $V, T$. Below $\Delta$ it has the same $V, T$-dependence and similar interference pattern, $\langle I\rangle \propto\left|W_{1} e^{i \kappa_{1}}+W_{2} e^{i \kappa_{2}}\right|^{2}$, as electron current in Eqs. (3) and (4) for $V, T<\Delta$. The relation between $\Delta$ and $T_{X}$ affects then only the $U$-dependence of the overall magnitude of the current, which increases or decreases with increasing $U$ in the electron and quasiparticle regimes, respectively. The two currents coincide at $\Delta \simeq T_{X}$.

In the case of the two-flux-pair variation, $m=2$, realized when $\nu_{0}=1 / 5$ and $\nu_{1}=1$, the Klein factors (11) anticommute and can be represented by Pauli matrices: $\bar{F}_{1,2}=\sigma_{1,2}$. In terms of the flux $n$ through the antidot, the Pauli matrices $\sigma$ act in the space of two states representing the parity of $n$. The low-energy scaling dimension of the quasiparticle tunneling operators $\bar{T}_{1,2}^{ \pm}$is equal to $-1 / 4$, the fact that makes Lagrangian (9) nonperturbative at low energies $V, T$. To understand this non-perturbative behavior, we notice that for $V, T<\Delta$, the quasiparticle terms $\bar{T}_{1,2}^{ \pm}$can be reduced to effective single-point tunneling

$$
\bar{T}_{1}^{+}+\bar{T}_{2}^{+}=(\Delta / 4 \pi) e^{i \frac{\lambda}{2} \vartheta(t)} \sum_{ \pm} X_{ \pm} e^{i \varphi_{ \pm}} \sigma_{ \pm},
$$

$$
X_{ \pm} \simeq(\Delta / D)^{2 / \lambda^{2}-1}\left[W_{1}^{2}+W_{2}^{2} \mp 2 W_{1} W_{2} \sin (\kappa / 2)\right]^{1 / 2},
$$

where $\varphi_{ \pm}=\arg \left[W_{1} \mp i W_{2} e^{-i \kappa / 2}\right]$, free bosonic field $\vartheta$ is defined by the correlator $\langle\vartheta(t) \vartheta(0)\rangle=g(0, t)$, and $\sigma_{ \pm}=\left(\sigma_{1} \pm i \sigma_{2}\right) / 2$. The model (13) can be mapped onto a model [14] of zero-energy resonant level in the Tomonage-Luttinger liquids (TLL) of interaction constant $g=1 / 3$. In this mapping, the amplitudes of tunneling into the level from the two TLL electrodes are $\sqrt{\Delta / 2 \pi} X_{ \pm}$, and our quasiparticle current coincides with the current through the level. Under conditions of resonance, $\kappa=2 \pi \times$ integer, the two amplitudes $X_{ \pm}$are equal, and the model (13) can be further reduced to a point scatterer in the uniform TLL of interaction strength $g^{-1} / 4=3 / 4$. The quasiparticle current $\langle I\rangle$ then is:

$$
\langle I\rangle=\left(\sigma_{0} V / 3\right)\left[1-G_{3 / 4}(2 V / 3 \Gamma, T / \Gamma)\right]
$$

where $\Gamma \propto \Delta X^{4} \simeq \Delta\left(T_{X} / \Delta\right)^{8 / 3}$, and $\sigma_{0}$ is the free electron conductance. The function $G_{g}$ is normalized TLL conductance and describes the cross-over from $G_{g}(v, 0) \simeq$ $\left(1-c_{1}(1 / g) v^{2(1 / g-1)}\right)$ for $v<1$ to $G_{g}(v, 0) \simeq c_{1}(g) v^{2(g-1)}$ for $v>1$ at zero temperature $T$, where $c_{1}(g)=\sqrt{\pi} \Gamma(g+$ $1) /(2 \Gamma(1 / 2+g))$, and similar dependence on $T$ at zero voltage $V$. The maximum conductance $\sigma_{0} / 3$ (14) is reached at $V, T \ll \Gamma$. The short-noise charge is equal to $2 / 3$ and is twice larger than the charge of one TLL quasiparticle, since only backscattering of pairs of quasiparticles is allowed at resonance. Small deviations of $\kappa$ from the resonant values restore backscattering of individual quasiparticles and lead to suppression of the tunnel current outside the resonances. The width of resonances changes with temperature as $(T / \Delta)^{2 / 3}$.

In conclusion, we have developed a theory of transport properties of antidot junction between two single-mode edges of different filling factors (Fig. 1). The theory predicts tunneling of quasiparticles of fractional statistics and charge $e / m$ set by variation $m$ of the number of flux pairs attached to electrons in the QHLs. The charge $e / m$ should be seen in the shot noise for weak interference between backscattering processes in the two point contacts of the junction, while quasiparticle statistics affects the tunnel current when the interference is strong.

This work was supported by the NSA and ARDA under ARO contract \# DAAD19-03-1-0126.
[1] R.B. Laughlin, Phys. Rev. Lett. 50, 1395 (1983).

[2] B.I. Halperin, Phys. Rev. Lett. 52, 1583 (1984); D. Arovas, J.R. Schrieffer, and F. Wilczek, Phys. Rev. Lett. 53, 722 (1984).

[3] C.L. Kane and M.P.A. Fisher, Phys. Rev. Lett. 72, 724
(1994).

[4] V.J. Goldman and B. Su, Science 267, 1010 (1995); V.J. Goldman, I. Karakurt, J. Liu, and A. Zaslavsky Phys. Rev. B 64, 085319 (2001).

[5] L. Saminadayar, D.C. Glattli, Y. Jin, and B. Etienne, 
Phys. Rev. Lett. 79, 2526 (1997); R. de-Picciotto, M. Reznikov, M. Heiblum, V. Umansky, G. Bunin, D. Mahalu, Nature 389, 162 (1997); M. Reznikov, R. de Picciotto, T.G. Griffiths, M. Heiblum, and V. Umansky, Nature 399, 238 (1999).

[6] E. Comforti, Y.C. Chung, M. Heiblum, V. Umansky, D. Mahalu, Nature 416, 515 (2002).

[7] I. Safi, P. Devillard, and T. Martin, Phys. Rev. Lett. 86, 4628 (2001); C.L. Kane, Phys. Rev. Lett. 90, 226802 (2003); S. Vishveshwara, Phys. Rev. Lett. 91, 196803 (2003).

[8] N.P. Sandler, C. de C. Chamon, and E. Fradkin, Phys. Rev. B 57, 12324 (1998); Phys. Rev. B 59, 12521 (1999).

[9] V.V. Ponomarenko and D.V. Averin, Phys. Rev. B 70, 195316 (2004).

[10] M.R. Geller, D. Loss, and G. Kirczenow, Phys. Rev. Lett.
77, 5110 (1996); M.R. Geller and D. Loss, Phys. Rev. B 56, 9692 (1997).

[11] J.K. Jain, Phys. Rev. Lett. 63, 199 (1989).

[12] V.V. Ponomarenko and D.V. Averin, JETP Lett. 74, 87 (2001); Phys. Rev. B 67, 35314 (2003).

[13] X.G. Wen, Phys. Rev. Lett. 64, 2206 (1990); J. Frölich and T. Kerler, Nucl. Phys. B 354, 369 (1991); M. Stone and M.P.A. Fisher, Int. J. Mod. Phys. B 8, 2539 (1994); N. Nagaosa and M. Kohmoto, Phys. Rev. Lett. 75, 4294 (1995).

[14] C.L. Kane and M.P.A. Fisher, Phys. Rev. B 46, 15233 (1992).

[15] C. Chamon and E. Fradkin, Phys. Rev. B 56, 2012 (1997).

[16] U. Weiss, Solid State Commun. 100, 281 (1996). 